\title{
Central corneal thickness in eyes with pseudoexfoliation syndrome in Kashmir Valley - a hospital based study
}

\author{
Sofi IA ${ }^{1}$, Nawaz $\mathbf{S}^{2}$, Wani JS ${ }^{3}$ \\ ${ }^{1}$ Dr Ishfaq Ahmad Sofi, MS. Registrar, Postgraduate Department of Ophthalmology, Government Medical College, \\ Srinagar, ${ }^{2}$ Dr Shah Nawaz, MD. Lecturer, Postgraduate Department of Ophthalmology, Government Medical College, \\ Srinagar, ${ }^{3}$ Dr Junaid S. Wani, MS. Associate Professor, Postgraduate Department of Ophthalmology, Government \\ Medical College, Srinagar, J\& K, India
}

Address for Correspondence: Dr Ishfaq Ahmad Sofi. Registrar, Postgraduate Department of Ophthalmology, Government Medical College Srinagar. Room no 402, Doctor's Hostel, opposite causality block, SMHS hospital, Karan Nagar, Srinagar, J\&K India

\begin{abstract}
Introduction: Pseudoexfoliation syndrome (PEX) is characterized by the production and accumulation of extracellular granular fibrillar material in many ocular tissues. Pseudoexfoliation has been closely associated with glaucoma and raised intraocular pressure (IOP) is the most important known risk factor for glaucoma. Central corneal thickness (CCT) may affect the accuracy of IOP measurements. Thus, it is possible to underestimate/overestimate the IOP reading in the PEX syndrome and overlook an early glaucomatous damage. The aim of our study was to determine the central corneal thickness (CCT) in eyes with pseudoexfoliation (PEX). Methods: Total 2076 eyes (of 1224 patients) with pseudoexfoliation on clinical examination were enrolled in this prospective clinical study and CCT was measured in each eye using specular microscopy. Results: Mean CCT $(\mu \mathrm{m})$ in eyes with Pseudoexfoliation was 525.43 \pm 34.27 . CCT in eyes with Hypertensive PEX $(513.2 \pm 27.8)$ and PEX Glaucoma $(509.22 \pm 29.76)$ was significantly thinner than in eyes with Normotensive PEX (528.17 \pm 30.33$)(\mathrm{P}=0.001, \mathrm{P}=0.0001$, respectively). There was no significant difference in CCT between the fellow eyes in cases of unilateral pseudoexfoliation $(\mathrm{P}=0.54)$. Conclusion: Mean central corneal thickness (CCT) in eyes with pseudoexfoliation is $525.4 \mu \mathrm{m}$ and CCT is significantly thinner in Hypertensive PEX as well as Glaucomatous PEX eyes than Normotensive PEX eyes.
\end{abstract}

Keywords: Central Corneal Thickness, Glaucoma, Pseudoexfoliation Syndrome.

\section{Introduction}

Pseudoexfoliation syndrome (PEX) is a systemic, age related Disorder [1], characterized by the production and accumulation of extracellular granular fibrillar material in many tissues and organs [2,3]. A typical sign of the PEX syndrome in the eye is white deposits accumulating on the pupillary border and on the anterior lens capsule, visible during an examination with the slit lamp [4]. Prevalence of PEX is $3.41 \%$ in South India and increases with age and baseline ocular hypertension (OHT) was a significant factor for conversion to glaucoma [5]. Grodum et al reported that glaucoma conversion rate was twice as high in patients with OHT and PEX (54 of 98 patients, 55.1\%) as in

Manuscript received: $4^{\text {th }}$ Sept 2015

Reviewed: $20^{\text {th }}$ Sept 2015

Author Corrected: $24^{\text {th }}$ Sept 2015

Accepted for Publication: $30^{\text {th }}$ Sept 2015 control patients (27 of 98 patients, $27.6 \%$ ) matched for age, sex, and intraocular pressure (IOP) without PEX after the mean of 8.7 years follow-up [6]. Accurate IOP measurement is an important factor in the diagnosis and management of glaucoma, but various sources of error may affect the accuracy of measurements. Central corneal thickness (CCT) is well known and affects the IOP readings [7]. Thus, it is possible to underestimate/overestimate the IOP reading in the PEX syndrome and overlook an early glaucomatous damage. There are conflicting reports about the CCT in patients with pseudoexfoliation syndrome (PEX) or pseudoexfoliation glaucoma (PEG). Most studies have found similar CCT in PEX eyes and normal eyes [8-11], although some authors have reported lower [12-14] or higher [15] values than the CCT of normal eyes. The aim of this study was to assess central corneal thickness 
(CCT) in patients with PEX syndrome with or without glaucoma using specular microscopy.

\section{Materials and Methods}

Place of Study: This study was conducted on the patients who attended General outdoor clinic of postgraduate department of ophthalmology, Government Medical College Srinagar between February 2013 \& August 2015. The study was approved by the ethical committee of our institute and followed the Declaration of Helsinki for research involving human subjects.

Inclusion and Exclusion Criteria: After proper informed consent a total 2076 eyes (of 1224 patients) with diagnosis of pseudoexfoliation syndrome were included in the study. Diagnosis of the syndrome was based on the typical slit-lamp appearance of pseudoexfoliative material at the anterior lens capsule and/or at the pupillary margin. Patients with history of ocular trauma, previous intraocular surgery or laser procedures, previous or present contact lens wear, early or suspect keratoconus were excluded.

Study Design: This was a prospective observational clinical study.

Sampling Method: Simple random sampling was done.

\section{Methods}

All the enrolled patients underwent a complete eye examination, including evaluation of visual acuity for distance and near vision using Snellen test-type, slit lamp biomicroscopy, intraocular pressure (IOP) measurements using Goldmann applanation tonometer (Zeiss AT 020 Applanation Tonometer; Carl Zeiss, Meditec AG, Jena, Germany), gonioscopy using a 4 mirror Sussmann lens (Volk Optical Inc., Mentor, OH), repeat slit lamp evaluation after dilatation for PEX deposits, detailed retinal examination with a binocular indirect ophthalmoscope using a +20 D lens, stereoscopic evaluation of the optic nerve head using a Volk aspheric +90 D lens at the slit lamp. All were asked to perform threshold visual field test using the Swedish interactive threshold algorithm standard 24-2 program (Humphrey Field Analyser, model 750i; Carl Zeiss Meditec AG, Jena Germany). In all patients, Central corneal thickness (CCT) was recorded by using specular microscope (Topcon SP-1P, Tokyo, Japan). Modern specular microscopes use advanced computer software and allows for the measuring of the central corneal thickness (pachymetry). The 2076 eyes (of 1224 patients) with PEX were subcategorized into Normotensive PEX, Hypertensive PEX, and PEX with glaucoma (PEG). Ocular hypertension was defined as an IOP greater than $21 \mathrm{~mm} \mathrm{Hg}$ with no glaucomatous defects on visual field testing and normal appearance of the optic disc. PEG patients had open-angle, glaucomatous optic disc, visual field changes and IOP greater than $21 \mathrm{~mm} \mathrm{Hg}$.

Statistical Methods: Statistical evaluation was performed using a computer software SPSS ( V. 12, Chicago, Ill ). The independent samples test ( $t$ test) was used for comparisons of means between the subgroups of PEX patients. A $p$ value of $<0.05$ was considered significant.

\section{Results}

The mean (SD) age was 71.5 (6.4) years in all PEX patients. Among all the 2076 eyes with PEX included in the study, 726 eyes were Normotensive PEX having IOP $\leq 20 \mathrm{mmHg}, 738$ eyes with Hypertensive PEX having IOP $\geq 21 \mathrm{mmHg}$ without glaucomatous changes and 612 eyes with PEG having IOP $\geq 21 \mathrm{mmHg}$ with glaucomatous changes. 372 patients had unilateral PEX. Demographic data and the mean (SD) central corneal thickness values in eyes with pseudoexfoliation syndrome along with mean IOP (SD) values are shown in Table 1. The mean (SD) CCT of all the patients with a PEX was $525.4(34.2) \mu \mathrm{m}$. In a subgroup analysis of PEX patients, the CCT was significantly thinner in Hypertensive PEX eyes $\{513(27.8)\}$ and PEG eyes $\{509(29.7)\}$ as compared to Normotensive PEX eyes $\{528.1(30.3)\}(\mathrm{P}=0.001$ and $\mathrm{P}=$ 0.0001 , respectively). Among the patients with unilateral PEX, there was statistically no difference in mean CCT between the PEX eye $(526.5 \pm 24.6 \mu \mathrm{m})$ and the fellow eye $(527.2 \pm 26.6 \mu \mathrm{m})(\mathrm{P}=0.54)$.

Table 1: Demographic data and Clinical characteristics. 


\begin{tabular}{|l|l|l|l|l|l|l|}
\hline \multicolumn{6}{|c|}{ Demographic data and central corneal thickness values in eyes with pseudoexfoliation syndrome with } \\
normal IOP, elevated IOP, and glaucoma
\end{tabular}

\section{Discussion}

Accurate measurement of IOP is an important factor in the diagnosis and management of glaucoma. It is well known that CCT varies widely among individuals. Furthermore, CCT affects the IOP measurements that are obtained with Goldmann applanation tonometer. Thus, it is possible to underestimate or overestimate the IOP readings and overlook an early glaucomatous damage. This could have serious clinical implication in PEX patients. The present study examined CCT in PEX eyes with or without glaucoma in Kashmir valley. We used specular microscopy to measure CCT because in many comparison studies specular microscopy has been defined to be more accurate and more reliable than the more common ultrasound pachymetry (USP) [16-18]. In our study we found that mean (SD) CCT was 525.4 (34.2) $\mu \mathrm{m}$ in the enrolled PEX eyes. The mean CCT in PEX eyes in patients with or without glaucoma has been reported as $493 \pm 33 \mu \mathrm{m}$ [12], $507 \pm 25 \mu \mathrm{m}$ [10], $524.2 \pm 21.3 \mu \mathrm{m}[9], 526.3 \pm 31.7 \mu \mathrm{m}[11], 528 \pm 30 \mu \mathrm{m}$ [15], $529 \pm 31 \mu \mathrm{m}$ [13], $529.7 \pm 30.3 \mu \mathrm{m}$ [19], and 530.7 $\mu \mathrm{m}[8], 531 \pm 7.3 \mu \mathrm{m}[14]$.

In a subgroup analysis of our study, mean (SD) CCT of Hypertensive PEX eyes $\{513.2(27.8)\}$ and PEG eyes $\{509.2(29.7)\}$ was significantly thinner as compared to Normotensive PEX eyes $\{528.1$ (30.3) $\}$. Tomaszewski BT et al used a specular microscope to assess CCT in PEX eyes and showed that the thinnest corneas occur in eyes of patients with secondary glaucoma with PEX $(508.2 \pm 32.6 \mu \mathrm{m})$ and this value is significantly different $(P<0.001)$ from the CCT of patients with PEX syndrome but without glaucoma $(529.7 \pm 30.3$ $\mu \mathrm{m})$ and from the CCT of people from the control group
$(527.7 \pm 29.4 \mu \mathrm{m})$ [19]. While as Kitsos et al used ultrasonic pachymeter for the assessment of CCT and showed that the lowest CCT value was obtained in patients with PEX glaucoma $(526.00 \pm 34.30 \mu \mathrm{m})$ and this was significantly thinner than the CCT of patients with PEX syndrome $(550.64 \pm 39.0 \mu \mathrm{m})$ and patients of the control group $(547.36 \pm 33.1 \mu \mathrm{m})(P<0.05)$ [20] Different data was presented by Inoue et al which showed that patients with PEX syndrome with glaucoma and without secondary glaucoma had thinner corneas $(529 \pm 31 \mu \mathrm{m})$ from the control group $(547 \pm$ $28 \mu \mathrm{m})(P=0.03)[13]$. At the same time they noticed that patients with secondary glaucoma and PEX had thicker corneas $(534 \pm 37 \mu \mathrm{m})$ than people with PEX without secondary glaucoma $(528 \pm 29 \mu \mathrm{m})$; however, results were not statistically significant $(P=0.68)$. The discrepancy in the results presented in relation to the conclusions of this study may be a consequence of a very small group of participants used as only 19 corneas of patients with PEX syndrome and only 7 with secondary glaucoma and PEX were included in their study. Shah et al proved that patients with glaucoma PEX had significantly thinner corneas than people with PEX without glaucoma $(530.7 \mu \mathrm{m}$ versus $553.9 \mu \mathrm{m})(P$ $<0.001)$ [8]. Interesting conclusions regarding the influence of PEX syndrome on the corneal stroma were included in the study published by Zheng et al in which the authors, using confocal microscopy, identified deposits of pseudoexfoliating materials in the cornea itself [21]. They also showed that the number of keratocytes in the corneal stroma of the eyes of patients with PEX syndrome (per unit of area) was smaller than in the group of people without PEX. They concluded 
that the presence of the pseudoexfoliating material induces apoptosis of corneal stroma keratocytes and in the end leads to the impoverishment of its extracellular structure. This may result in the thinning of the cornea in PEX eyes.

\section{Conclusions}

We concluded that among the people belonging to Kashmir valley, mean central corneal thickness (CCT) in eyes with pseudoexfoliation is $525.4 \mu \mathrm{m}$ and PEX is mostly seen in older age group. The CCT is significantly thinner in Hypertensive PEX as well as Glaucomatous PEX eyes than Normotensive PEX eyes. Further studies are needed to clarify the clinical importance of our findings and ascertain whether there is a correlation between CCT and pseudoexfoliation.

\section{Funding: Nil \\ Conflict of interest: None. \\ Permission of IRB: Yes}

\section{References}

1. Ritch R, Schlotzer-Schrehardt U. Exfoliation syndrome. Survey of Ophthalmology 2001;45(4):265$315 . \quad$ DOI: http://dx.doi.org/10.1016/S00396257(00)00196-X

2. Schumacher S, Schlotzer-Schrehardt U, Martus P, Lang W, Naumann GO. Pseudoexfoliation syndrome and aneurysms of the abdominal aorta. Lancet 2001 Feb;357(9253):359-60.

3. Schlotzer-Schrehardt UM, Koca MR, Naumann GOH, Volkholz H. Pseudoexfoliation syndrome: ocular manifestation of a systemic disorder?. Arch Ophthalmol. 1992 Dec;110(12):1752-6. doi:10.1001/archopht.1992.01080240092038.

4. Naumann GOH, Schlotzer-Schrehardt U. Keratopathy in pseudoexfoliation syndrome as a cause of corneal endothelial decompensation. Ophthalmology 2000 Jun;107(6):1111-24. doi:10.1016/S01616420(00)00087-7.

5. Vijaya L, Asokan R, Panday M, Choudhari NS, Sathyamangalam RV, Velumuri L et al. The Prevalence of Pseudoexfoliation and the Long-term Changes in Eyes With Pseudoexfoliation in a South Indian Population. J Glaucoma 2015 May;00:000-007. doi: 10.1097/IJG.0000000000000276.
6. Grodum K, Heijl A, Bengtsson B. Risk of glaucoma in ocular hypertension with and without pseudoexfoliation. Ophthalmology 2005 Mar;112(3):386-90.

7. Doughty MJ, Zaman ML. Human corneal thickness and its impact on intraocular pressure measures: a review and metaanalysis approach. Survey of Ophthalmology $2000 \quad$ Mar;44(5):367-408. doi:10.1016/S0039-6257(00)00110-7.

8. Shah S, Chatterjee A, Mathai M, Kelly SP, Kwartz J, Henson D et al. Relationship between corneal thickness and measured intraocular pressure in a general ophthalmology clinic. Ophthalmology 1999 Nov;106(11):2154-60. doi:10.1016/S01616420(99)90498-0.

9. Ventura AC, Bohnke M, Mojon DS. Central corneal thickness measurements in patients with normal tension glaucoma, primary open angle glaucoma, pseudoexfoliation glaucoma, or ocular hypertension. $\mathrm{Br}$ J Ophthalmol. 2001 Jul;85(7):792-5.

10. Detorakis ET, Koukoula S, Chrisohoou F, Konstas AG, Kozobolis VP. Central corneal mechanical sensitivity in pseudoexfoliation syndrome. Cornea. 2005 Aug;24(6):688-91.

11. Yagci R, Eksioglu U, Midillioglu I, Yalvac I, Altiparmak E, Duman S. Central corneal thickness in primary open angle glaucoma, pseudoexfoliative glaucoma, ocular hypertension, and normal population. Eur J Ophthalmol. 2005 May-Jun;15(3):324-8.

12. Bechmann M, Thiel MJ, Roesen B, Ullrich S, Ulbig MW, Ludwig K. Central corneal thickness determined with optical coherence tomography in various types of glaucoma. Br J Ophthalmol. 2000 Nov;84(11):1233-7.

13. Inoue $\mathrm{K}$, Okugawa $\mathrm{K}$, Oshika $\mathrm{T}$, Amano $\mathrm{S}$. Morphological study of corneal endothelium and corneal thickness in pseudoexfoliation syndrome. Jpn J Ophthalmol. 2003 May-Jun;47(3):235-9.

14. Aghaian E, Choe JE, Lin S, Stamper RL. Central corneal thickness of Caucasians, Chinese, Hispanics, Filipinos, African Americans, and Japanese in a glaucoma clinic. Ophthalmology 2004 Dec;111(12):2211-9. 
15. Puska P, Vasara K, Harju M, Setala K. Corneal thickness and corneal endothelium in normotensive subjects with unilateral exfoliation syndrome. Graefes Arch Clin Exp Ophthalmol. 2000 Aug;238(8):659-63.

16. Tai L, Khaw K, Ng C, Subrayan V. Central corneal thickness measurements with different imaging devices and ultrasound pachymetry. Cornea 2013 Jun;32(6):766-71.

17. Almubrad TM, Osuagwu UL, AlAbbadi I, Ogbuehi KC. Comparison of the precision of the Topcon SP$3000 \mathrm{P}$ specular microscope and an ultrasound pachymeter. Clin Ophthalmol. 2011;5:871-6. doi: 10.2147/OPTH.S21247.

18. Ogbuehi KC, Osuagwu UL. Repeatability and interobserver reproducibility of Artemis-2 highfrequency ultrasound in determination of human corneal thickness. Clin Ophthalmol. 2012; 6: 761-9. doi: 10.2147/OPTH.S31690.
19. Tomaszewski BT, Zalewska R, Mariak Z. Evaluation of the Endothelial Cell Density and the Central Corneal Thickness in Pseudoexfoliation Syndrome and Pseudoexfoliation Glaucoma. Journal of Ophthalmology 2014;2014:123683. doi: $10.1155 / 2014 / 123683$.

20. Kitsos G, Gartzios C, Asproudis I, Bagli E. Central corneal thickness in subjects with glaucoma and in normal individuals (with or without pseudoexfoliation syndrome). Clin Ophthal. 2009;3:537-42.

21. Zheng X, Shiraishi A, Okuma S, Mizoue S, Goto T, Kawasaki $S$ et al. In vivo confocal microscopic evidence of keratopathy in patients with pseudoexfoliation syndrome. Invest Ophthalmol Vis Sci. 2011 Mar;52(3):1755-61. doi: 10.1167/iovs.106098.

\section{How to cite this article?}

Sofi IA, Nawaz S, Wani JS. Central corneal thickness in eyes with pseudoexfoliation syndrome in Kashmir Valley - a hospital based study. Int J Med Res Rev 2015;3(9):934-938. doi: 10.17511/ijmrr.2015.i9.174. 